\title{
Mathematica Bohemica
}

\section{Murat Diker}

The counterparts of some cardinal functions in bitopological spaces. I.

Mathematica Bohemica, Vol. 120 (1995), No. 3, 237-245

Persistent URL: http://dml.cz/dmlcz/126011

\section{Terms of use:}

(C) Institute of Mathematics AS CR, 1995

Institute of Mathematics of the Czech Academy of Sciences provides access to digitized documents strictly for personal use. Each copy of any part of this document must contain these Terms of use.

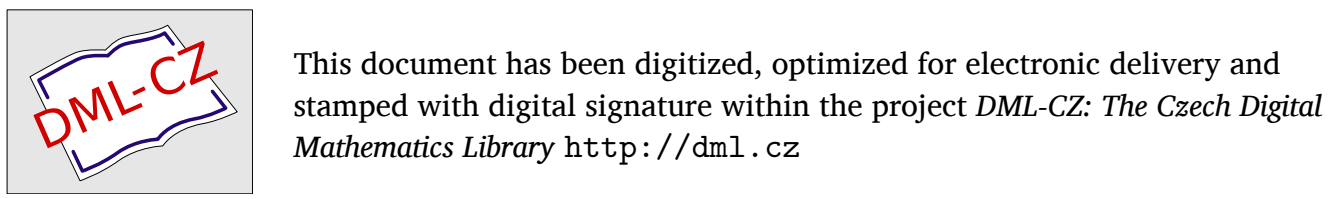




\section{THE COUNTERPARTS OF SOME CARDINAL FUNCTIONS IN BITOPOLOGICAL SPACES I}

Murat Diker, Ankara

(Received December 8, 1993)

Summary. This is the first in a series of papers aimed at defining and studying bitopological counterparts of the principal cardinal invariants in topology. It is devoted to study of analogues of the functions weight, density and cellularity.

Keywords: bidensity, quasi-regular density, bicellularity

AMS classification: $54 \mathrm{~A} 25,54 \mathrm{E} 55$

The basic motivation for the introduction of topological invariants is the classification of topological spaces. For this reason, cardinal functions play a very important role in General Topology [3] and [4].

While it is possible to study the cardinal functions of the two topologies of a bitopological space separately, this is not necessarily the most appropriate course, since it is often the interrelationship between these topologies which is significant in a bitopological space setting. For example, a bitopological space might satisfy a significant separation axiom, without either of the topologies doing so. Since this will have repercussions on the relations between the various cardinal functions, it is clearly of interest to try and develop cardinal functions which are directly related to the bitopological structure, and this has been our aim.

The approach of Kopperman and Meyer [5], whose paper came to the attention of the author after the completion of this work, is somewhat different. Their idea is essentially to associate with a bitopological space the largest value of a given (topological) cardinal function for the two topologies and for the joint topology. For some cardinal functions, including the weight in particular, this leads to a function with a natural bitopological interpretation, but for a large class of cardinal functions it reduces to the consideration of that function for the joint topology alone, and a 
good deal of bitopological structure can be lost in the passage to the joint topology. In the analogues of density and cellularity presented here, we have tried to involve the bitopological structure in a more essential way, and have attempted to find relations between these invariants which depend on bitopological rather than topological properties.

Throughout this paper, $(X, u, v)$ will denote a bitopological space, and $u \vee v$ the joint topology on $X$. If $\varphi$ is a (topological) cardinal function, $\varphi_{u}(X), \varphi_{v}(X)$ and $\mathrm{j} \varphi(X)$ will denote the values of $\varphi$ for the spaces $(X, u),(X, v)$ and $(X, u \vee v)$ respectively.

Let us recall that $(X, u, v)$ is called:

(i) weakly pairwise $\mathrm{T}_{0}$ if given $x, y \in X$ with $x \neq y$, there exists $G \in u \cup v$ such that $x \in G, y \notin G$ or $x \notin G, y \in G$;

(ii) weakly pairwise $\mathrm{T}_{2}$ if given $x, y \in X$ with $x \neq y$, there exist $U \in u, V \in v$ with $U \cap V=\emptyset$ and $x \in U, y \in V$ or $y \in U, x \in V$;

(iii) pairwise $\mathrm{T}_{2}$ if given $x, y \in X$ with $x \neq y$, there exist $U \in u, V \in v$ with $U \cap V=\emptyset$ and $x \in U, y \in V$

(iv) pairwise regular if given $F, u$-closed ( $v$-closed) and $x \notin F$, there exist $U \in u$, $V \in v$ with $U \cap V=\emptyset$ and $x \in U, F \subseteq V(x \in V, F \subseteq U)$. [6]

The material in this series that forms part of the author's Ph.D. Thesis [2] was written under the supervision of Dr. L. M. Brown.

\section{BIWEIGH'T}

1.1. Definition. Let $(X, u, v)$ be a bitopological space, $d$ an open dual family, that is $d \subseteq u \times v$. The family $d$ is called a bibase for $X$ if it satisfies the following condition:

$\forall(G, H) \in u \times v$

$$
x \in G \cap H \Rightarrow(\exists(U, V) \in d)(x \in U \subseteq G \text { and } x \in V \subseteq H)
$$

The cardinal number

$$
\mathrm{bw}(X)=\min \{|d|: d \text { is a bibase for } X\}
$$

is called the biweight of the space $(X, u, v)$.

The following theorem is easily proved and shows that our definition of a biweight is equivalent to that given in [5]. 


\subsection{Theorem.}

$$
\mathrm{jw}(X) \leqslant \mathrm{bw}(X)=\max \left\{\mathrm{w}_{u}(X), \mathrm{w}_{v}(X)\right\} .
$$

The joint weight is not always equal to the biweight, as the following example shows:

1.3. Example. We take $X=\mathbb{N}$. Let $\mathscr{F}$ be a free ultrafilter on $X$, that is $\cap \mathscr{F}=\emptyset$. We consider the topologies $u=\mathscr{F} \cup\{\emptyset\}$ and $v=$ discrete on $X$. It can be easily seen that

$$
\mathrm{jw}(X)=\omega<\mathrm{bw}(X)=\omega_{1} .
$$

1.4. Theorem. If $(X, u, v)$ is weakly pairwise $\mathbf{T}_{0}$, then

$$
|X| \leqslant \exp b w(X)
$$

Proof. It is an immediate consequence of the fact that $X$ is jointly $\mathrm{T}_{0}$ and jw $(X) \leqslant \operatorname{bw}(X)$.

\section{BIDENSITY}

2.1. Definition. Let $(X, u, v)$ be a bitopological space. A subset $A$ of $X$ is called bidense in $X$ [1] if it is both $u$-dense and $v$-dense. The cardinal number

$$
\operatorname{bd}(X)=\min \{|A|: A \text { is a bidense subset of } X\}
$$

is called the bidensity of the space $(X, u, v)$.

\subsection{Theorem.}

$$
\max \left\{\mathrm{d}_{u}(X), \mathrm{d}_{v}(X)\right\}=\operatorname{bd}(X) \leqslant \mathrm{jd}(X)
$$

We can find a bitopological space with $\operatorname{bd}(X)<\mathrm{jd}(X)$. Hence, our density function is different from that given in [5]

2.3. Example. Let $u$ be the topology with the base $\{(-\infty, a]: a \in \mathbb{R}\}$, and $v$ the topology with the base $\{[b, \infty): b \in \mathbb{R}\}$ on $\mathbb{R}$. The bitopological space $(\mathbb{R}, u, v)$ is jointly discrete and so $\mathrm{jd}(\mathbb{R})=\omega_{1}$. The set of rational numbers $\mathbb{Q}$ is bidense in $\mathbb{R}$, so that $\operatorname{bd}(\mathbb{R})=\omega$. Hence, we obtain $b d(\mathbb{R})<\mathrm{jd}(\mathbb{R})$. Now let us recall the following two well known results in topological spaces: Let $(X, \tau)$ be a topological space. 
(i) If $(X, \tau)$ is regular, then $w(X) \leqslant \operatorname{expd}(X)$.

(ii) If $(X, \tau)$ is $\mathrm{T}_{2}$, then $\mathrm{w}(X) \leqslant \exp ^{3} \mathrm{~d}(X)$.

For a bitopological space $(X, u, v)$, if both $u$ and $v$ are regular topologies, the analogue of result (i) namely,

$$
\mathrm{bw}(X) \leqslant \exp b \mathrm{~d}(X)
$$

is easily verified.

However, we do not have the same result for pairwise regular spaces:

2.4. Example. Let $\gamma \geqslant \omega$ be a cardinal number and $\alpha=\cup\left\{\exp ^{n} \gamma: n \in \omega\right\}$. Consider the topologies

$$
u=\{[0, \eta): \eta<\alpha\} \cup\{\alpha, 0\}, v=\{[\beta, \alpha): \beta<\alpha\} \cup\{0\}
$$

on $X=[0, \alpha)=\alpha$. It is easy to show that the space $(X, u, v)$ is pairwise regular. Furthermore, the set $S=\left\{\exp ^{n} \gamma: n \in \omega\right\} \cup\{0\}$ is bidense in $X$ and $\omega=|S|=$ $\mathrm{bd}(X)<\alpha$. Since $\alpha$ is a strong limit cardinal and $\mathrm{bw}(X)=\alpha$, we find

$$
\operatorname{expbd}(X)<\operatorname{bw}(X) .
$$

Remaining with this space, we see that $(X, u, v)$ is weakly pairwise $\mathrm{T}_{2}$. The cardinal number $\alpha$ is strong limit cardinal, and so

$$
\exp ^{3} \operatorname{bd}(X)<\mathrm{bw}(X) \text {. }
$$

This means that the analogue of result (ii) is not true for weakly pairwise $T_{2}$ spaces. The following example shows that this result does not hold for pairwise $\mathrm{T}_{2}$ spaces either:

2.5. Example. Let $X=\omega_{4}=\left[0, \omega_{4}\right)$. We consider topologies $u$ and $v$ with the bases

$$
\begin{gathered}
\{\{\gamma\}: \gamma \leqslant \omega\} \bigcup\left\{([0, \omega) \backslash A) \cup\{\eta\}: \omega<\eta<\omega_{4},|A|<\omega, A \subset \omega\right\} \\
\bigcup\{[0, \omega) \backslash B:|B|<\omega, B \subset \omega\}
\end{gathered}
$$

and

$$
\{\{\gamma\}: \gamma \leqslant \omega\} \bigcup\left\{\left(\omega, \omega_{4}\right) \backslash A:|A|<\omega, A \subset\left(\omega, \omega_{4}\right)\right\},
$$

respectively. It is easy to check that $X$ is pairwise $\mathrm{T}_{2}$. Let $A \subset\left(\omega, \omega_{4}\right)$ and $|A|=\omega$. The set $C=[0, \omega) \cup A$ is bidense in $X$, and $|C|=\operatorname{bd}(X)$. Since $\operatorname{bw}(X)=\omega_{4}$, $\operatorname{bd}(X)<\operatorname{bw}(X)$. Then we have

$$
\omega_{3}=\exp ^{3} \operatorname{bd}(X)<\operatorname{bw}(X)=\omega_{4} .
$$




\section{REgUlaR AND QUASI-REgULAR DENSITY}

In view of the above negative results, we seek to define new density functions which are better behaved in this respect.

3.1. Definition. Let $(X, u, v)$ be a bitopological space and $A$ a bidense subset of $X . A$ is called regularly (quasi-regularly) dense in $X$ if there exists a $u$ base $\delta_{u}$ and a $v$ base $\delta_{v}$ satisfying the conditions

$$
\forall U, U^{\prime} \in \delta_{u}, U \cap A=U^{\prime} \cap A \Rightarrow{\overleftarrow{U^{v}}}^{v}={\overline{U^{\prime}}}^{v}
$$

and (or)

$$
\forall V, V^{\prime} \in \delta_{v}, V \cap A=V^{\prime} \cap A \Rightarrow \bar{V}^{u}={\overline{V^{\prime}}}^{u}
$$

The cardinal number

$$
\operatorname{rd}(X)=\min \{|A|: A \text { is regularly dense in } X\}
$$

will be called the regular density of $(X, u, v)$. Similarly, the quasi-regular density of $X$ can be defined as the cardinal number

$$
\operatorname{qrd}(X)=\min \{|A|: A \text { is quasi-regularly dense in } X\}
$$

It is easy to verify

\subsection{Theorem.}

$$
\operatorname{bd}(X) \leqslant \operatorname{qrd}(X) \leqslant \operatorname{rd}(X) \leqslant \mathrm{jd}(X) .
$$

3.3. Example. Consider the space $(X, u, v)$ of Example 2.3, and the bases $\delta_{u}=\{(-\infty, a]: a \in \mathbb{R}\}, \delta_{v}=\{[b, \infty): b \in \mathbb{R}\}$ of $u$ and $v$ respectively. The set of rational numbers $\mathbb{Q}$ is regularly dense in $X$ : If $[a, \infty) \cap \mathbb{Q}=[b, \infty) \cap \mathbb{Q}$ and $(-\infty, c] \cap \mathbb{Q}=(-\infty, d] \cap \mathbb{Q}$, then $a=b$ and $c=d$. It follows that

$$
\operatorname{bd}(X)=\operatorname{rd}(X)=\operatorname{qrd}(X)<\operatorname{jd}(X) .
$$

3.4. Ex a mple. We take the space $(X, u, v)$ of Example 2.5 and consider the bidense set $C$ of $X$. It is easy to check that $C$ is not quasi-regularly dense in $X$. Furthermore, if $A$ is a bidense subset of $X$ and $|A|=\omega_{2}$ or $\omega_{3}$, then it cannot be quasi-regularly dense. Hence, we have

$$
\operatorname{bd}(X)<\operatorname{qrd}(X)=\operatorname{rd}(X)=\mathrm{jd}(X) .
$$


3.5. Example. Let $X=\left[0, \omega_{2}\right)=\omega_{2}$. Consider the topology $u$ on $X$ with the base

$$
\left\{[0, \omega) \cup\{\eta\}: \omega<\eta<\omega_{2}\right\} \bigcup\{0, \omega\} \bigcup\{\{\gamma\}: \gamma \leqslant \omega\}
$$

and the topology $v$ with the base

$$
\{\{0, \gamma\}: \gamma<\omega\} \bigcup\left\{\left(\omega_{2} \backslash \omega_{1}\right) \cup\{\omega\}, \omega_{1},\{\omega\},\{0\}, 0\right\}
$$

Clearly, $A=[0, \omega]$ is bidense in $X$, and $|A|=\operatorname{bd}(X)$. It can be easily seen that $A$ is not quasi-regularly dense in $X$. Now we take the set $B=\left[0, \omega_{1}\right] . B$ is bidense, but not regularly dense. However, $B$ is quasi-regularly dense and $|B|=\omega_{1}=\operatorname{qrd}(X)$. It follows easily that

$$
\operatorname{bd}(X)<\operatorname{qrd}(X)<\operatorname{rd}(X)=\mathrm{jd}(X) .
$$

We may now present

3.6. Theorem. If $(X, u, v)$ is pairwise regular, then

$$
\operatorname{bw}(X) \leqslant \exp \operatorname{rd}(X)
$$

Proof. Let $A$ be regularly dense in $X,|A|=\operatorname{rd}(X)$. Consider the bases $\delta_{u}$ and $\delta_{v}$ corresponding to $A$. Clearly, $d=\delta_{u} \times \delta_{v}$ is a bibase for $X$. Let $d=\left\{\left(U_{\alpha}, V_{\alpha}\right)\right.$ : $\alpha \in \Gamma\}$. It is easy to see that

$$
d^{\prime}=\left\{\left(\text { int }_{u}{\overline{U_{\alpha}}}^{v}, \text { int }_{v}{\overline{V_{\alpha}}}^{u}\right): \alpha \in \Gamma\right\}
$$

is also a bibase for $X$. For each $\alpha \in \Gamma$, take

$$
E_{\alpha}=A \cap U_{\alpha}, F_{\alpha}=A \cap V_{\alpha}
$$

Consider $\alpha, \beta \in \Gamma, \alpha \neq \beta$, with $\left(E_{\alpha}, F_{\alpha}\right)=\left(E_{\beta}, F_{\beta}\right)$. Since $A$ is regularly dense $\left(\bar{U}_{\alpha}^{v}, \bar{V}_{\alpha}^{u}\right)=\left(\bar{U}_{\beta}^{v}, \bar{V}_{\beta}^{u}\right)$, we have $\left(\right.$ int $_{u} \bar{U}_{\alpha}^{v}$, int $\left._{v} \bar{V}_{\alpha}^{u}\right)=\left(\right.$ int $_{u} \bar{U}_{\beta}^{v}$, int $\left._{u} \bar{V}_{\beta}^{u}\right)$. Hence, we find

$$
\operatorname{bw}(X) \leqslant\left|d^{\prime}\right| \leqslant\left|\left\{\left(E_{\alpha}, F_{\alpha}\right): \alpha \in \Gamma\right\}\right| \leqslant|\mathscr{P}(A)|=\exp \operatorname{rd}(X) .
$$

3.7. Theorem. If $(X, u, v)$ is weakly pairwise $\mathrm{T}_{2}$, then

$$
|X| \leqslant \exp ^{2} \operatorname{qrd}(X) .
$$


Proof. Let $A$ be quasi-regularly dense in $X,|A|=\operatorname{qrd}(X)$, and let $\delta_{u}$ and $\delta_{v}$ be bases of $u, v$, respectively, with

$$
\forall U, U^{\prime} \in \delta_{u}, U \cap A=U^{\prime} \cap A \Rightarrow \bar{U}^{v}={\overline{U^{\prime}}}^{v}
$$

or

$$
\forall V, V^{\prime} \in \delta_{v}, V \cap A=V^{\prime} \cap A \Rightarrow \bar{V}^{u}={\overline{V^{\prime}}}^{u}
$$

Assume that (1) holds. For each $x \in X$ consider the family

$$
A_{x}=\left\{G \cap A: x \in G \in \delta_{u} \cup \delta_{v}\right\} .
$$

Consider $x, y \in X$ with $x \neq y$. We will show that $A_{x} \neq A_{y}$. Suppose that $A_{x}=A_{y}$. Since $X$ is weakly pairwise $\mathrm{T}_{2}$, there exist $U \in \delta_{u}$ and $V \in \delta_{v}$ such that $U \cap V=\emptyset$ and $x \in U, y \in V$ or $x \in V, y \in U$. If $x \in U, y \in V$, clearly $U \cap A \in A_{x}$. Since $A_{x}=A_{y}$ there exists $U^{\prime} \in \delta_{u}$ or $V^{\prime} \in \delta_{v}$ such that

$$
y \in U^{\prime}, U^{\prime} \cap A=U \cap A
$$

or

$$
y \in V^{\prime}, V^{\prime} \cap A=V \cap A
$$

Assume that (3) holds. Since $U \cap V=\emptyset$, we have $U \subseteq X \backslash V$ and so $\bar{U}^{v} \subseteq X \backslash V$. By (1), ${\overline{U^{\prime}}}^{v} \subseteq X \backslash V$, that is $U^{\prime} \cap V=\emptyset$. But this contradicts $y \in U^{\prime} \cap V$. Now assume that (4) holds. Then $\emptyset=(U \cap A) \cap(V \cap A)=\left(V^{\prime} \cap A\right) \cap(V \cap A)=V^{\prime} \cap V \cap A$. Since A is $v$-dense, we deduce $V^{\prime} \cap V=\emptyset$, which contradicts $y \in V^{\prime} \cap V$. If we suppose $x \in V, y \in U$, we obtain a similar contradiction. Thus $x \neq y$ implies that $A_{x} \neq A_{y}$. If we consider the property (2), then we obtain the same result. Hence, we have

$$
|X|=\left|\left\{A_{x}: x \in X\right\}\right| \leqslant|\mathscr{P}(\mathscr{P}(A))|=\exp ^{2} \operatorname{qrd}(X) .
$$

3.8. Corollary. If $(X, u, v)$ is weakly pairwise $\mathbf{T}_{2}$, then

$$
\operatorname{bw}(X) \leqslant \exp ^{3} \operatorname{qrd}(X) .
$$

Clearly, $\operatorname{rd}(X)$ and $\operatorname{qrd}(X)$ may be replaced by $\mathrm{jd}(X)$ in the above results. However, the examples show that in general the given inequalities are sharper. 


\section{Bicellularity}

We now turn to the question of defining cellularity in a bitopological setting.

4.1. Definition. Let $(X, u, v)$ be a bitopological space and $\mathscr{C}=\left\{\left(U_{\alpha}, V_{\alpha}\right)\right.$ : $\alpha \in \Gamma\}$ an open dual family in $X$. The family $\mathscr{C}$ is said to be a bicellular in $X$ if

(i) $\forall \alpha \in \Gamma, U_{\alpha} \cap V_{\alpha} \neq \emptyset$

(ii) $\alpha, \beta \in \Gamma, \alpha \neq \beta \Rightarrow U_{\alpha} \cap V_{\beta}=\emptyset$ or $U_{\beta} \cap V_{\alpha}=\emptyset$.

The cardinal number

$$
\mathrm{bc}(X)=\sup \{|\mathscr{C}|: \mathscr{C} \text { is a bicellular family in } X\}
$$

is called the bicellularity of $(X, u, v)$.

Clearly, we have

\subsection{Theorem.}

$$
\mathrm{bc}(X) \leqslant \mathrm{jc}(X)
$$

4.3. Example. Let $X=\left[0, \omega_{1}\right)=\omega_{1}$, let $u$ be the topology with the base

$$
\{\{\gamma\}: \gamma \leqslant \omega\} \bigcup\left\{[0, \omega) \cup\{\eta\}: \eta \in\left(\omega, \omega_{1}\right)\right\} \bigcup\{\omega\}
$$

and $v$ the topology with the base

$$
\{\{0, \gamma\}: \gamma \in \omega\} \bigcup\left\{\omega_{1} \backslash \omega,\{0\}, 0\right\}
$$

It can be easily seen that, for the space $(X, u, v)$

$$
\omega=\operatorname{bc}(X)<\mathrm{jc}(X)=\omega_{1}
$$

For a topological space, it is trivial to show that $c(X) \leqslant \mathrm{d}(X)$. However, a similar result is not true for the cardinal invariants bicellularity, bidensity, regular density and quasi-regular density.

Consider the space $(X, u, v)$ of Example 2.3. The dual family $\mathscr{C}=\{((-\infty, a],[a, \infty))$ : $a \in \mathbb{R}\}$ is a bicellular family and $|\mathscr{C}|=b c(X)=\omega_{1}$, and so we obtain

$$
\omega=\mathrm{bd}(X)=\operatorname{qrd}(X)=\operatorname{rd}(X)<\mathrm{bc}(X) .
$$

However, we have the following results:

\subsection{Theorem}

$$
\operatorname{bc}(X) \leqslant \min \{\operatorname{jd}(X), \exp \operatorname{qrd}(X)\} .
$$


Proof. $\quad \mathrm{bc}(X) \leqslant \mathrm{jd}(X)$ is immediate by Theorem 4.2. Now we show that $\operatorname{bc}(X) \leqslant \exp \operatorname{qrd}(X)$. Let $\mathscr{C}=\left\{\left(U_{\alpha}, V_{\alpha}\right): \alpha \in \Gamma\right\}$ be a bicellular family and $A$ a quasi-regularly dense subset of $X$ with $|A|=\operatorname{qrd}(X)$. We denote by $\delta_{u}$ and $\delta_{v}$, respectively, the $u$ base and $v$ base corresponding to $A$. Without loss of generality, we can assume that the elements of $\mathscr{C}$ belong to $\delta_{u} \times \delta_{v}$. Now $\alpha, \beta \in \Gamma, \alpha \neq \beta$, implies $U_{\alpha} \cap A \neq U_{\beta} \cap A$ or $V_{\alpha} \cap A \neq V_{\beta} \cap A$. Indeed, assume $U_{\alpha} \cap A=U_{\beta} \cap A$ and $V_{\alpha} \cap A=V_{\beta} \cap A$. Then by the quasi-regular density of $A$, we have $\bar{U}_{\alpha}{ }^{v}=\bar{U}_{\beta}{ }^{v}$ or $\bar{V}_{\alpha}{ }^{u}=\bar{V}_{\beta}{ }^{u}$. Let $\bar{U}_{\alpha}{ }^{v}=\bar{U}_{\beta}{ }^{v}$. Then $\bar{U}_{\alpha}{ }^{v} \cap V_{\beta} \neq \emptyset$ and $\bar{U}_{\beta}{ }^{v} \cap V_{\alpha} \neq \emptyset$. Hence, we have $U_{\alpha} \cap V_{\beta} \neq \emptyset$ and $U_{\beta} \cap V_{\alpha} \neq \emptyset$. But this contradicts bicellularity of $\mathscr{C}$. If $\bar{V}_{\alpha}{ }^{u}=\bar{V}_{\beta}{ }^{v}$, we obtain a similar contradiction. Hence,

$$
|\mathscr{C}|=\left|\left\{\left(U_{\alpha} \cap A, V_{\alpha} \cap A\right): \alpha \in \Gamma\right\}\right| \leqslant|\mathscr{P}(A \times A)|=\exp \operatorname{qrd}(X) .
$$

Finally, we find $\mathrm{bc}(X) \leqslant \exp \operatorname{qrd}(X)$.

4.5. Theorem. Let $(X, u, v)$ be a bitopological space and $A$ a jointly dense subset of $X$. Then

$$
\mathrm{bc}(A)=\mathrm{bc}(X) .
$$

Proof. Let $\mathscr{C}$ be a bicellular family in $X$. Clearly, the family $\{(U \cap A, V \cap A)$ : $(U, V) \in \mathscr{C}\}$ is a bicellular family in $A$. Hence, bc $(X) \leqslant \mathrm{bc}(A)$. Now let $\mathscr{H}$ be a bicellular family in $A$. For each $(G, H) \in \mathscr{H}$, we can find $\left(U_{G}, V_{H}\right) \in u \times v$ such that $G=U_{G} \cap A, H=V_{H} \cap A$. If we use the joint density of $A$, we can easily see that $\left\{\left(U_{G}, V_{H}\right):(G, H) \in \mathscr{H}\right\}$ is a bicellular family in $X$, and so bc $(A) \leqslant \mathrm{bc}(X)$.

In view of Example 3.3, the joint density of $A$ in Theorem 4.5 cannot be replaced by bidensity, quasi-regular density or regular density, because the set of rational numbers $\mathbb{Q}$ is bidense, quasi-regularly dense and regularly dense, but $b c(\mathbb{Q})=\omega<b c(X)$.

\section{References}

[1] L. M. Brown: Dual Covering Theory, Confluence Structures and the Lattice of Bicontinuous Functions. Ph.D.Thesis Univ. of Glasgow, 1980.

[2] M. Diker: Cardinal Functions in Bitopological spaces. Ph. D. Thesis Univ. of Hacettepe, Ankara, 1991

[3] 1. Juhász: Cardinal Functions in Topology. Math. Center Tracts No. 34, Math. Centrum, Amsterdam, 1971.

[4] 1. Juhász: Cardinal Functions in Topology Ten Years Later. Math. Center Tracts No. 123, Math. Centrum, Amsterdam, 1980.

[5] R. D. Kopperman, P. R. Meyer: Cardinal invariants of bitopological spaces. Casopis pro pěstování matematiky 114 (1989), no. 4, 374-380.

[6] M. J. Saegrove: Pairwise complete regularity and compactification in bitopological spaces. J. London Math. Soc. 7(1973), no. 2, 286-290.

Author's address: Murat Diker, Department of Science Education, Hacettepe University, Beytepe, 06532 Ankara, Türkiye. 